TRANSACTIONS OF THE

AMERICAN MATHEMATICAL SOCIETY

Volume 350, Number 12, December 1998, Pages 4919-4930

S $0002-9947(98) 02373-3$

\title{
KÄHLER DIFFERENTIALS, THE T-FUNCTOR, AND A THEOREM OF STEINBERG
}

\author{
W. G. DWYER AND C. W. WILKERSON
}

\begin{abstract}
Let $T$ be the functor on the category of unstable algebras over the Steenrod algebra constructed by Lannes. We use an argument involving Kähler differentials to show that $T$ preserves polynomial algebras. This leads to new and relatively simple proofs of some topological and algebraic theorems.
\end{abstract}

\section{InTRODUCTION}

Fix a prime number $p$, and let $\mathcal{K}$ denote the category of unstable algebras over the $\bmod p$ Steenrod algebra $\mathcal{A}_{p}[19, \S 1]$. Recall that an elementary abelian p-group is a finite abelian group which is a vector space over $\mathbf{F}_{p}$. Given an object $R$ of $\mathcal{K}$ and an elementary abelian $p$-group $E$, Lannes [16] constructs an associated "function object" $T_{E}(R)$; if $X$ is a space and $R=\mathrm{H}^{*}\left(X ; \mathbf{F}_{p}\right)$, then $T_{E}(R)$ is an algebraic approximation to the mod $p$ cohomology of the space of maps $\mathrm{B} E \rightarrow X$. More generally, associated to any $\mathcal{K}$-map $f: R \rightarrow \mathrm{H}^{*}\left(\mathrm{~B} E ; \mathbf{F}_{p}\right)$ is a "component" $T_{E, f}(R)$ of $T_{E}(R)$ (see $\left.[16,2.5]\right)$; for $R=\mathrm{H}^{*}\left(X ; \mathbf{F}_{p}\right)$ this is an algebraic approximation to the $\bmod p$ cohomology of an appropriate component of the space of maps $\mathrm{B} E \rightarrow X$.

Our main theorem is the following one.

1.1 Theorem. Suppose that $R$ is an object of $\mathcal{K}$ which as an algebra is a connected finitely generated polynomial algebra over $\mathbf{F}_{p}$. Then for any elementary abelian $p$ group $E$ and $\mathcal{K}$-map $f: R \rightarrow \mathrm{H}^{*}\left(\mathrm{~B} E ; \mathbf{F}_{p}\right), T_{E, f}(R)$ is also a finitely generated polynomial algebra over $\mathbf{F}_{p}$.

The proof of 1.1 proceeds by combining a characterization of graded polynomial algebras in terms of Kähler differentials with a study of how the functor $T_{E}$ interacts with the process of constructing the Kähler differentials.

Theorem 1.1 leads pretty directly to three other results. If $K$ is a subgroup of the group $G$, let $C_{G}(K)$ denote the centralizer in $G$ of $K$.

1.2 Theorem. Suppose that $G$ is a compact Lie group with $\mathrm{H}^{*}\left(\mathrm{~B} G ; \mathbf{F}_{p}\right)$ a polynomial algebra over $\mathbf{F}_{p}$. Then for any elementary abelian p-subgroup $E$ of $G$, $\mathrm{H}^{*}\left(\mathrm{~B} C_{G}(E) ; \mathbf{F}_{p}\right)$ is also a polynomial algebra over $\mathbf{F}_{p}$.

Proof. Suppose that $E$ is an elementary abelian $p$-subgroup of $G$ and that

$$
f: \mathrm{H}^{*}\left(\mathrm{~B} G ; \mathbf{F}_{p}\right) \rightarrow \mathrm{H}^{*}\left(\mathrm{~B} E ; \mathbf{F}_{p}\right)
$$

Received by the editors August 1, 1996.

1991 Mathematics Subject Classification. Primary 55N99, 13D99.

The authors were supported in part by the National Science Foundation.

(C)1998 American Mathematical Society 
is the map induced by the inclusion $E \rightarrow G$. By [15], $\mathrm{H}^{*}\left(\mathrm{~B} C_{G}(E) ; \mathbf{F}_{p}\right)$ is isomorphic as an object of $\mathcal{K}$ to $T_{E, f}\left(\mathrm{H}^{*}\left(\mathrm{~B} G ; \mathbf{F}_{p}\right)\right)$. The theorem follows from 1.1.

Parallel to this is a theorem about $p$-compact groups [11]. If $h: Y \rightarrow X$ is a homomorphism of $p$-compact groups [11,3.1], let $C_{X}(h(Y))$ denote the centralizer of $h(Y)$ in $X[11,3.5]$.

1.3 Theorem. Suppose that $X$ is a p-compact group with $\mathrm{H}^{*}\left(\mathrm{~B} X ; \mathbf{F}_{p}\right)$ a polynomial algebra over $\mathbf{F}_{p}$. Then for any elementary abelian $p$-group $E$ and homomorphism $h: E \rightarrow X, \mathrm{H}^{*}\left(\mathrm{~B} C_{X}(h(E)) ; \mathbf{F}_{p}\right)$ is also a polynomial algebra over $\mathbf{F}_{p}$.

Proof. By definition, $\mathrm{BC}_{X}(h(E))$ is the component of the space of maps from $\mathrm{B} E$ to $\mathrm{B} X$ containing the map $\mathrm{B} h$. Denote by $f: \mathrm{H}^{*}\left(\mathrm{~B} X ; \mathbf{F}_{p}\right) \rightarrow \mathrm{H}^{*}\left(\mathrm{~B} E ; \mathbf{F}_{p}\right)$ the map induced by $\mathrm{B} h$. According to $\left[12\right.$, pf. of 8.1], $\mathrm{H}^{*}\left(\mathrm{~B} C_{X}(h(E)) ; \mathbf{F}_{p}\right)$ is isomorphic to $T_{E, f}\left(\mathrm{H}^{*}\left(\mathrm{~B} X ; \mathbf{F}_{p}\right)\right)$. The theorem follows from 1.1 .

Suppose that $k$ is a field and that $V$ is a finite-dimensional vector space over $k$. We let $V^{\#}$ denote the dual of $V$, and $\operatorname{Sym}\left(V^{\#}\right)$ the symmetric algebra on $V^{\#}$. If $k$ is infinite, $\operatorname{Sym}\left(V^{\#}\right)$ is the algebra of polynomial functions on $V$; in any case, $\operatorname{Sym}\left(V^{\#}\right)$ is a polynomial algebra over $k$ isomorphic to $k\left[x_{1}, \ldots, x_{d}\right]$, where $d=$ $\operatorname{dim}_{k}(V)$. Any group $W$ of automorphisms of $V$ acts on $\operatorname{Sym}\left(V^{\#}\right)$ in a natural way and has an associated fixed subalgebra $\operatorname{Sym}\left(V^{\#}\right)^{W}$. The following is a theorem of Nakijima [18, 2.1.2]. See [14] for a version that applies to the case in which the action of $W$ on $V$ is irreducible, as well as for many examples. We give another proof in $\S 5$.

1.4 Theorem. Suppose that $V$ is a finite dimensional vector space over the field $\mathbf{F}_{p}$ and that $W$ is a subgroup of Aut $(V)$. Let $U$ be a subset of $V$, and $W_{U}$ the subgroup of $W$ consisting of elements which fix $U$ pointwise. Then if $\operatorname{Sym}\left(V^{\#}\right)^{W}$ is a polynomial algebra over $\mathbf{F}_{p}$, so is $\operatorname{Sym}\left(V^{\#}\right)^{W_{U}}$.

Reflection groups and Steinberg's theorem. Both 1.2 and 1.4 have characteristic zero versions which are consequences of the following theorem of Steinberg. Recall that if $V$ is a finitely generated free module over a domain $k$, an element $w$ of finite order in $\operatorname{Aut}_{k}(V)$ is said to be a reflection if image $\left(w-\operatorname{Id}_{V}\right)$ has rank 1. (Sometimes such a $w$ is called a generalized reflection or a pseudoreflection, and the word reflection is reserved for the case in which the order of $w$ is 2 . We will use the word reflection in the wider sense.) A subgroup $W \subset \operatorname{Aut}_{k}(V)$ is said to be generated by reflections (or said to be a reflection group) if it is generated by the reflections that it contains.

1.5 Theorem (Steinberg, [22, Thm. 1.5]). Suppose that $k$ is a field of characteristic zero, and that $W \subset G L(n, k)$ is a finite group generated by reflections. Let $U$ be a subset of $k^{n}$ and $W_{U}$ the subgroup of $W$ consisting of elements which fix $U$ pointwise. Then $W_{U}$ is also generated by reflections.

The tie connecting 1.5 to 1.2 and 1.4 is the following classical result.

1.6 Theorem. [8] [21] [7] [20] [5] [2] Suppose that $V$ is a finite dimensional vector space over a field $k$ of characteristic zero, and that $W \subset \operatorname{Aut}(V)$ is a finite group. Then $\operatorname{Sym}\left(V^{\#}\right)^{W}$ is a polynomial algebra over $k$ if and only if $W$ is generated by reflections. 
Remark. If the field $k$ in 1.6 has finite characteristic and $\operatorname{Sym}\left(V^{\#}\right)^{W}$ is a polynomial algebra, then $W$ is generated by reflections [20], but the converse does not necessarily hold (see for instance [23]).

Here are the characteristic zero versions of 1.2 and 1.4.

1.7 Theorem. Suppose that $G$ is a compact Lie group with the property that $\mathrm{H}^{*}(\mathrm{~B} G ; \mathbf{Q})$ is a polynomial algebra over $\mathbf{Q}$. Let $T^{\prime}$ be a toral subgroup of $G$. Then $\mathrm{H}^{*}\left(\mathrm{~B} C_{G}\left(T^{\prime}\right) ; \mathbf{Q}\right)$ is also a polynomial algebra.

Remark. If $G$ is a connected compact Lie group, then $\mathrm{H}^{*}(\mathrm{~B} G ; \mathbf{Q})$ is always a polynomial algebra.

Proof of 1.7. Suppose that $K$ is a compact Lie group with maximal torus $T_{K}$ and Weyl group $W_{K}=\operatorname{Norm}_{K}\left(T_{K}\right) / T_{K}$. Let $V_{K}=\mathbf{Q} \otimes \pi_{1} T_{K}$; the group $W_{K}$ acts by conjugation on $T_{K}$ and hence on $V_{K}$. Recall that the obvious restriction map gives an isomorphism

$$
\mathrm{H}^{*}(\mathrm{~B} K ; \mathbf{Q}) \cong \mathrm{H}^{*}\left(\mathrm{~B} T_{K} ; \mathbf{Q}\right)^{W_{K}} \cong \operatorname{Sym}\left(V_{K}^{\#}\right)^{W_{K}} .
$$

Now let $T_{G}$ be a maximal torus of $G$. By adjusting $T^{\prime}$ up to conjugacy we can assume that $T^{\prime} \subset T_{G}$. Let $V=V(G)$; by 1.8 and 1.6 , the image of the map $W(G) \rightarrow \operatorname{Aut}(V)$ is generated by reflections. Let $U \subset V$ be the image of $\pi_{1} T^{\prime}$ and let $W_{U} \subset W(G)$ denote the subgroup of elements which fix $U$ pointwise. By 1.5, the image of the map $W_{U} \rightarrow \operatorname{Aut}(V)$ is generated by reflections. It is easy to identify $W_{U}$ with the Weyl group of $C_{G}\left(T^{\prime}\right)$, and it follows from another application of 1.8 and 1.6 that $\mathrm{H}^{*}\left(\mathrm{~B} C_{G}\left(T^{\prime}\right) ; \mathbf{Q}\right)=\operatorname{Sym}\left(V^{\#}\right)^{W_{U}}$ is a polynomial algebra.

1.9 Theorem. Suppose that $V$ is a finite dimensional vector space over $\mathbf{Q}$, and that $W \subset \operatorname{Aut}(V)$ is a finite group. Let $U$ be a subset of $V$, and $W_{U}$ the subgroup of $W$ consisting of elements which fix $U$ pointwise. Then if $\operatorname{Sym}\left(V^{\#}\right)^{W}$ is a polynomial algebra over $\mathbf{Q}$, so is $\operatorname{Sym}\left(V^{\#}\right)^{W_{U}}$.

Proof. This is a direct combination of 1.5 and 1.6. The theorem also holds if $\mathbf{Q}$ is replaced by any other field of characteristic zero.

In some sense, then, 1.2 and 1.1 can be viewed as generalizations of Steinberg's Theorem 1.5 to finite characteristic. We give the following relatively simple example to show that some adjustment in the statement of the theorem is necessary in order to obtain such a generalization.

1.10 Examples. There is a rank 3 free abelian subgroup $\Sigma$ of $S L(5, \mathbb{Z})$ and an element $u \in(\mathbb{Z})^{5}$ such that for any prime $p$ the following condition holds: the mod $p$ reduction $\bar{\Sigma}$ of $\Sigma$ acts faithfully on $\left(\mathbf{F}_{p}\right)^{5}$ as a group generated by reflections, but the stabilizer in $\bar{\Sigma}$ of the $\bmod p$ reduction of $u$ is a subgroup of order $p$ not generated by reflections.

Organization of the paper. For the rest of the paper, $p$ denotes a fixed prime number. Section 2 describes a way to recognize polynomial algebras over $\mathbf{F}_{p}$ by looking at modules of Kähler differentials. Section 3 shows that Lannes's functor $T$ preserves Kähler differentials, and $\S 4$ shows that $T$ preserves the properties of these differentials which characterize polynomial algebras. The proof of 1.1 is immediate. In $\S 5$ there is an explanation of how to obtain 1.4 from 1.1. Finally, $\S 6$ gives a construction of Example 1.10. 
Acknowledgments. The authors thank L. Avramov for discussions on the commutative algebra points in section 2. The formulation of Theorem 2.3 was prompted by a more general conjecture mentioned in a talk by $\mathrm{C}$. Weibel. We note that Campbell-Huges-Shank [6] have independently found examples with properties similar to 1.10 .

\section{KäHLER DIFFERENTIALS AND POLYNOMIAL ALGEBRAS}

We first need a recognition principle for polynomial algebras. If $R$ is a commutative algebra over a commutative base ring $k$, there is a short exact sequence of $R \otimes_{k} R$-modules

$$
0 \rightarrow J \rightarrow R \otimes_{k} R \stackrel{\mu}{\longrightarrow} R \rightarrow 0
$$

where the map $\mu$ is multiplication on $R$. The two inclusions $R \rightarrow R \otimes_{k} 1$ and $R \rightarrow 1 \otimes_{k} R$ give possibly different left $R$-module structures on $J$.

2.1 Definition. The module of Kähler differentials of $R$ relative to $k$, denoted $\Omega(R \mid k)$, is $J / J^{2}=J / \operatorname{image}\left(J \otimes_{k} J \stackrel{\mu}{\longrightarrow} J\right)$.

2.2 Remark. The action of $R \otimes_{k} R$ on $\Omega(R \mid k)$ factors through the multiplication map $\mu$, and so the above two $R$-module structures on $J$ induce the same $R$-module structure on $\Omega(R \mid k)$. Let $S=R \otimes_{k} R$. The module $\Omega(R \mid k)$ can be identified with $J \otimes_{S} R$ or equivalently, by a long exact sequence argument, with $\operatorname{Tor}_{1}^{S}(R, R)$.

There is a map $d: R \rightarrow \Omega(R \mid k)$ which is a universal $k$-linear derivation and sends $x \in R$ to the equivalence class of $x \otimes 1-1 \otimes x$. If $R$ has a set $\left\{x_{1}, \ldots, x_{n}\right\}$ of $k$ algebra generators, $\Omega(R \mid k)$ is generated as an $R$-module by $\left\{d x_{1}, \ldots, d x_{n}\right\}$; if $R$ is a polynomial algebra $k\left[x_{1}, \ldots, x_{n}\right]$, then $\Omega(R \mid k)$ is the free $R$-module on the classes $d x_{i}$. Under some conditions a converse of this last observation holds.

2.3 Theorem. Let $k$ be a perfect field of characteristic $p$ and $R$ a finitely generated connected graded algebra over $k$. Suppose that $\Omega(R \mid k)$ is a free $R$-module, and that $R$ has no nilpotent elements. Then $R$ is isomorphic to a polynomial algebra $k\left[x_{1}, \ldots, x_{n}\right]$.

Remark. The integer $n$ which appears in 2.3 equals $\operatorname{rank}_{R}(\Omega(R \mid k))$. The assumption that $R$ has no nilpotent elements cannot be removed from 2.3. To see this, note that if $R$ is a connected graded bicommutative Hopf algebra over $k$, a standard untwisting argument gives an isomorphism

$$
\operatorname{Tor}_{*}^{R \otimes_{k} R}(R, R) \cong R \otimes_{k} \operatorname{Tor}_{*}^{R}(k, k) .
$$

In particular, $\Omega(R \mid k)$ is free as an $R$-module. Now take $R$ to be, say, a primitively generated Hopf algebra with one even dimensional generator $x$ truncated at height $p$. Then $\Omega(R \mid k)$ is free as an $R$-module but $R$ is not a polynomial algebra.

Proof of 2.3. This follows from the Jacobian Criterion for smoothness; see for example [17, Th. 30.3] or [13, Cor. 16.22]. The particular case we have is simpler than the general one and so we include an elementary proof. Since $R$ is finitely generated and connected, there exists a surjection $\phi: S=k\left[x_{1}, \ldots, x_{n}\right] \rightarrow R$, where the $x_{i}$ are polynomial variables of positive grading. Choose $\phi$ in a minimal way so that the induced map on indecomposable quotients is an isomorphism. Let $I$ be the kernel of $\phi$; the goal is to show that $I$ is zero. Assume not, select a nonzero element $f \in I$ of minimal grading $m>0$, and renumber the variables $x_{i}$ if necessary so that 
$\left\{x_{i}\right\}_{i=1}^{n_{0}}$ is the set of variables of grading less than $m$. Since $\phi$ is an isomorphism on indecomposable quotients, $f$ can be written as a polynomial $f\left(x_{1}, \ldots, x_{n_{0}}\right)$.

The map $\phi: S \rightarrow R$ is an isomorphism in gradings below $m$, as are the induced maps $\Omega(S \mid k) \rightarrow \Omega(R \mid k)$ and $k \otimes_{S} \Omega(S \mid k) \rightarrow k \otimes_{R} \Omega(R \mid k)$. Now the elements $\left\{d x_{i}\right\}_{i=1}^{n_{0}}$ have linearly independent images in $k \otimes_{S} \Omega(S \mid k)$, and hence the elements $\left\{d \phi\left(x_{i}\right)\right\}_{i=1}^{n_{0}}$ are linearly independent in $k \otimes_{R} \Omega(R \mid k)$. This implies that $\left\{d \phi\left(x_{i}\right)\right\}_{i=1}^{n_{0}}$ is part of an $R$-basis for $\Omega(R \mid k)$.

Since $\phi(f)$ vanishes, so does $d \phi(f)$, and we get an equation

$$
0=d \phi(f)=\sum_{i=1}^{n_{0}} \phi\left(\partial f / \partial x_{i}\right) d \phi\left(x_{i}\right) .
$$

As above, though, the elements $\left\{d \phi\left(x_{i}\right)\right\}_{i=1}^{n_{0}}$ are linearly independent over $R$, so the equation implies that $\phi\left(\partial f / \partial x_{i}\right)$ vanishes for $i \leq n_{0}$. Since $\partial f / \partial x_{i}$ is of grading less than $m$ we conclude, from the choice of $m$, that $\partial f / \partial x_{i}$ vanishes for $i \leq n_{0}$ and hence for all $i$ (because $f$ only depends on $\left\{x_{i}\right\}_{i=1}^{n_{0}}$ ). Since $k$ is perfect and $S$ is a polynomial algebra, there exists a polynomial $g \in S$ such that $f=(g)^{p}$, and, because $R$ contains no nilpotent elements, $g$ must also be in the kernel of $\phi$. But $g$ has grading $m / p<m$, and so the existence of $g$ contradicts the assumption that $\phi$ is an isomorphism in gradings below $m$.

\section{KÄHLER DIFFERENTIALS AND $T$}

As in $[19, \S 1]$, let $\mathcal{A}_{p}$ be the $\bmod p$ Steenrod algebra, $\mathcal{U}$ the category of unstable modules over $\mathcal{A}_{p}$, and $\mathcal{K}$ the category of unstable algebras over $\mathcal{A}_{p}$. An object of $\mathcal{U}$ or of $\mathcal{K}$ is a nonnegatively graded $\mathbf{F}_{p}$-vector space with an action of $\mathcal{A}_{p}$ and, in the case of $\mathcal{K}$, a graded commutative multiplication which obeys the Cartan formula and a $p$ th power condition $[19,1.3,1.4]$. There is a forgetful functor $\mathcal{K} \rightarrow \mathcal{U}$. If $X$ is a space, the cohomology algebra $\mathrm{H}^{*}\left(X ; \mathbf{F}_{p}\right)$, with its usual cup product structure, belongs to $\mathcal{K}$.

Let $E$ be an elementary abelian $p$-group and $\mathrm{B} E$ its classifying space, Lannes has studied the functor $T_{E}: \mathcal{U} \rightarrow \mathcal{U}$ which is left adjoint to the functor which sends $M \in \mathcal{U}$ to $M \otimes_{\mathbf{F}_{p}} \mathrm{H}^{*}\left(\mathrm{~B} E ; \mathbf{F}_{p}\right)$ (note that the action of $\mathcal{A}_{p}$ on such a tensor product is given by the Cartan formula). The functor $T_{E}$ has some remarkable algebraic properties; in particular, it is exact, preserves tensor products over $\mathbf{F}_{p}$ up to natural isomorphism, and lifts to an identically named functor $\mathcal{K} \rightarrow \mathcal{K}[19, \S 3]$. The topological significance of $T_{E}$ has to do with its usefulness for computing the cohomology of function spaces, but we are interested in it from an algebraic point of view.

There is a slight refinement of $T_{E}$ which has topological applications in computing the cohomology of individual components of function spaces. Suppose that $R \in \mathcal{K}$, and that $f: R \rightarrow \mathrm{H}^{*}\left(\mathrm{~B} E ; \mathbf{F}_{p}\right)$ is a $\mathcal{K}$-map. By adjointness $f$ corresponds to a $\mathcal{K}$-map $f^{b}: T_{E}(R) \rightarrow \mathbf{F}_{p}$. Since the range of $f^{b}$ is concentrated in degree $0, f^{b}$ amounts to an ordinary ring homomorphism $f^{b}: T_{E}^{0}(R) \rightarrow \mathbf{F}_{p}$; this homomorphism makes $\mathbf{F}_{p}$ into a module over $T_{E}^{0}(R)$. Indeed, it follows from the fact that $T_{E}^{0}(R)$ is a $p$-Boolean algebra $[19,3.8]$ that $\mathbf{F}_{p}$ is a flat module over $T_{E}^{0}(R)$. We define $T_{E, f}(R)=\mathbf{F}_{p} \otimes_{T_{E}^{0} R} T_{E}(R)$. More generally, let $\mathcal{U}(R)$ be the category in which an object is an element $M \in \mathcal{U}$ together with a $\mathcal{U}$ map $R \otimes_{\mathbf{F}_{p}} M \rightarrow M$ which makes $M$ into a module over $R$. If $M \in \mathcal{U}(R)$ then $T_{E}(M) \in \mathcal{U}\left(T_{E}(R)\right)$ (this follows from the fact that $T_{E}$ preserves tensor products) 
and we define $T_{E, f} M=\mathbf{F}_{p} \otimes_{T_{E}^{0} R} T_{E} M \cong T_{E, f}(R) \otimes_{T_{E} R} T_{E} M$. The construction $T_{E, f}$ gives an exact functor $\mathcal{U}(R) \rightarrow \mathcal{U}\left(T_{E, f} R\right)$. In particular, $T_{E, f}(R)$ is flat as a module over $T_{E} R$.

Remark. Suppose that $\operatorname{Hom}_{\mathcal{K}}\left(R, \mathrm{H}^{*}\left(\mathrm{~B} E ; \mathbf{F}_{p}\right)\right)$ is a finite set; this is always the case if $R$ is finitely generated as an algebra (since $\mathrm{H}^{*}\left(\mathrm{~B} E ; \mathbf{F}_{p}\right)$ is finite in each dimension). In this situation $T_{E}^{0}(R)$ is isomorphic as a ring to a direct product of copies of $\mathbf{F}_{p}$, indexed by the $\mathcal{K}$-maps $f: R \rightarrow \mathrm{H}^{*}\left(\mathrm{~B} E ; \mathbf{F}_{p}\right)$. There is a corresponding product decomposition $[19,3.8 .6]$

$$
T_{E} R \cong \prod_{f} T_{E, f} R .
$$

For the rest of this section we assume that $E$ is a fixed elementary abelian $p$ group and write $T$ for $T_{E}$ and $T_{f}$ for $T_{E, f}$. The goal of this section is to prove the following two propositions.

3.1 Proposition. Let $R$ be an object of $\mathcal{K}$ and $f: R \rightarrow \mathrm{H}^{*}\left(\mathrm{~B} E ; \mathbf{F}_{p}\right)$ a $\mathcal{K}$-map. Then there is a natural isomorphism of $T_{f} R$-modules

$$
T_{f} \Omega\left(R \mid \mathbf{F}_{p}\right) \cong \Omega\left(T_{f} R \mid \mathbf{F}_{p}\right)
$$

3.2 Remark. An object $R$ of $\mathcal{K}$ need not be commutative as a ring (in general it is graded commutative), but we follow 2.2 in defining $\Omega\left(R \mid \mathbf{F}_{p}\right)$ as $\operatorname{Tor}_{1}^{S}(R, R)$, where $S=R \otimes \mathbf{F}_{p} R$. It will become clear below how to identify this as a object of $\mathcal{U}(R)$.

Note that if $R \in \mathcal{K}$ is commutative for the transparent reason that $p=2$ or that $R$ is concentrated in even degrees, then $T(R)$ and hence any $T_{f}(R)$ are commutative for the same reason $[19,3.6]$.

An algebra $R$ is said to be nilpotent free if the only nilpotent element of $R$ is the zero element.

3.3 Proposition. Let $R$ be an object of $\mathcal{K}$ which is nilpotent free. Then for any $\mathcal{K}$-map $f: R \rightarrow \mathrm{H}^{*}\left(\mathrm{~B} E ; \mathbf{F}_{p}\right), T_{f} R$ is nilpotent free.

The proof of 3.1 depends on two lemmas.

3.4 Lemma. Let $R$ and $S$ be objects of $\mathcal{K}, g: S \rightarrow R$ and $f: R \rightarrow \mathrm{H}^{*}\left(\mathrm{~B} E ; \mathbf{F}_{p}\right) a$ pair of $\mathcal{K}$-maps with $g$ surjective, and $M$ an object of $\mathcal{U}(R)$. Let $h=f g$. Then $T_{h} M$ is naturally isomorphic to $T_{f} M$ as an object of $\mathcal{U}\left(T_{h} S\right)$.

Proof. Observe that in the statement of $3.4, M$ is considered to be an $S$-module via the homomorphism $g: S \rightarrow R$, and $T_{f} M$ is a $T_{h} S$-module via the induced homomorphism $T_{h} S \rightarrow T_{f} R$.

The adjoint $h^{\text {b }}$ of $h$ is the composite $T(S) \stackrel{T(g)}{\longrightarrow} T(R) \stackrel{f^{b}}{\longrightarrow} \mathbf{F}_{p}$. Since $g$ is surjective and $T$ is exact, $T(g)$ is also surjective and in particular the map $T^{0}(S) \rightarrow T^{0}(R)$ induced by $T(g)$ is surjective. It is now clear that for any $T^{0}(R)$-module $N$ (such as $T(M))$ the map $\mathbf{F}_{p} \otimes_{T^{0} S} N \rightarrow \mathbf{F}_{p} \otimes_{T^{0} R} N$ is an isomorphism, and the lemma follows.

Note that if $R$ is an object of $\mathcal{K}$ and $M, N$ are objects of $\mathcal{U}(R)$, then for any $i \geq 0$ the $R$-module $\operatorname{Tor}_{i}^{R}(M, N)$ is also in a natural way an object of $\mathcal{U}(R)$; the $\mathcal{A}_{p}$ action on these $R$-modules can be obtained, for instance, by letting $\mathcal{A}_{p}$ act via the Cartan formula on the bar construction $[19,6.4]$. 
3.5 Lemma. Suppose that $R$ is an object of $\mathcal{K}, f: R \rightarrow \mathrm{H}^{*}\left(\mathrm{~B} E ; \mathbf{F}_{p}\right)$ is a $\mathcal{K}$-map, and $M, N$ are objects of $\mathcal{U}(R)$. Then there are natural isomorphisms in $\mathcal{U}\left(T_{f} R\right)$ :

$$
T_{f}\left(\operatorname{Tor}_{i}^{R}(M, N)\right) \cong \operatorname{Tor}_{i}^{T_{f} R}\left(T_{f} M, T_{f} N\right), \quad i \geq 0 .
$$

Proof. Since $T$ is exact and preserves tensor products, it follows as in $[19,6.4 .2]$ that there are natural isomorphisms $T\left(\operatorname{Tor}_{i}^{R}(M, N)\right) \cong \operatorname{Tor}_{i}^{T R}(T M, T N)$ in $\mathcal{U}(T R)$. Since $T_{f}(R)$ is flat (in fact projective [19, pf. of 6.4.3]) as a $T(R)$-module, there are natural isomorphisms

$$
T_{f}(R) \otimes_{T R} \operatorname{Tor}_{i}^{T R}(T M, T N) \cong \operatorname{Tor}_{i}^{T R}\left(T_{f} M, T N\right) .
$$

Again by flatness there is an isomorphism between $\operatorname{Tor}_{i}^{T R}\left(T_{f} M, T N\right)$ and $\operatorname{Tor}_{i}^{T_{f} R}\left(T_{f} M, T_{f} N\right)$ (cf. [19, pf. of 6.4.3]). Combining these isomorphisms gives the desired result.

Proof of 3.1. Let $S=R \otimes_{\mathbf{F}_{p}} R$ and let $h: S \rightarrow \mathrm{H}^{*}\left(\mathrm{~B} E ; \mathbf{F}_{p}\right)$ be the composite of $f$ with the multiplication map $S \rightarrow R$. By 3.5, there is a natural isomorphism $T_{h}\left(\operatorname{Tor}_{1}^{S}(R, R)\right) \cong \operatorname{Tor}_{1}^{T_{h} S}\left(T_{h} R, T_{h} R\right)$. By 3.4 there are natural isomorphisms $T_{h}\left(\operatorname{Tor}_{1}^{S}(R, R)\right) \cong T_{f}\left(\operatorname{Tor}_{1}^{S}(R, R)\right)$ and $T_{h}(R) \cong T_{f}(R)$. Finally, by using the fact that $T$ preserves tensor products over $\mathbf{F}_{p}$ we obtain an isomorphism

$$
T_{h}(S)=T(S) \otimes_{T^{0} S} \mathbf{F}_{p} \cong T_{f}(R) \otimes_{\mathbf{F}_{p}} T_{f}(R) .
$$

To finish up, identify $\Omega\left(R \mid \mathbf{F}_{p}\right)$ with $\operatorname{Tor}_{1}^{S}(R, R)(3.2)$.

Proof of 3.3. Suppose that $R$ is an object of $\mathcal{K}$ which is nilpotent free. We first show that $T R$ is nilpotent free. Since $R$ is graded commutative, it is clear that either $R$ is concentrated in even degrees, or $p=2$. Let $\Phi(R)$ be the object of $\mathcal{U}$ constructed as in $[19,1.7 .2]$ by multiplying the degrees of elements in $R$ by a factor of $p$. There is a natural $\mathcal{U}$-map $\lambda_{R}: \Phi(R) \rightarrow R$ which loosely speaking sends each element of $R$ to its $p$ th power. Since $R$ is nilpotent free, the map $\lambda_{R}$ is a monomorphism. By $[19,3.4]$, the map $\lambda_{T R}$ can be identified with $T\left(\lambda_{R}\right)$. Since $T$ is exact, it follows that $\lambda_{T R}$ is a monomorphism and hence that $T R$ is nilpotent free.

The final step is to show that $T_{f}(R)$ is nilpotent free. The functors $T$ and $T_{f}$ commute with colimits (because $T$ is a left adjoint and $T_{f}$ is obtained from $T$ by a tensor product). This implies that it is enough to treat the case in which $R$ is a finitely generated object of $\mathcal{K}$, i.e., generated by a finite number of elements under product, sum, and the operation of $\mathcal{A}_{p}$. In this case there are only a finite number of $\mathcal{K}$-maps $R \rightarrow \mathrm{H}^{*}\left(\mathrm{BE} ; \mathbf{F}_{p}\right)$ and $T^{0}(R)$ is isomorphic as a ring to a direct product of copies of $\mathbf{F}_{p}$, one for each such $\mathcal{K}$-map [19,3.8.6]. It follows that $T_{f}(R)$ is a direct factor, as a ring, of $T(R)$, and so $T_{f}(R)$ is nilpotent free if $T(R)$ is.

\section{Free $R$ MOdules ANd $T$}

We continue using the notation of the previous section in letting $E$ stand for an elementary abelian $p$-group and writing $T$ and $T_{f}$ for $T_{E}$ and $T_{E, f}$ respectively. Our goal is to complete the proof of 1.1 by showing that $T_{f}$ preserves the freeness of modules, in the following sense.

4.1 Proposition. Suppose that $R$ is an object of $\mathcal{K}$ and that $M$ is object of $\mathcal{U}(R)$ which is free as an $R$-module. Then for any $\mathcal{K}$-map $f: R \rightarrow \mathrm{H}^{*}\left(\mathrm{~B} E ; \mathbf{F}_{p}\right), T_{f}(M)$ is free as a $T_{f}(R)$-module. 
Proof of 1.1. This consists of stringing together 3.1, 4.1, 3.3, and 2.3. Note that $T_{f}(R)$ is finitely generated as a polynomial algebra because in general $T$ preserves finite generation of algebras $[10,1.4]$.

Proof of 4.1. Since $T_{f}(R)$ is a connected graded algebra, in order to show that $T_{f}(M)$ is a free module over $T_{f}(R)$ it is enough to show that $\operatorname{Tor}_{i}^{T_{f} R}\left(\mathbf{F}_{p}, T_{f} M\right)$ vanish for $i>0$ (actually, vanishing of Tor ${ }_{1}$ would be enough). Let $H=\mathrm{H}^{*}\left(\mathrm{~B} E ; \mathbf{F}_{p}\right)$. The map $f^{b}: T_{f}(R) \rightarrow \mathbf{F}_{p}$ that is used in computing Tor is adjoint to $f: R \rightarrow H$. It follows from naturality that $f^{b}$ is the composite of $T_{f}(f): T_{f}(R) \rightarrow T_{f}(H)$ with the map $\iota^{b}: T_{f}(H) \rightarrow \mathbf{F}_{p}$ adjoint to the identity map $\iota: H \rightarrow H$. In particular, for any $T_{f}(R)$-module $N$ there is a natural isomorphism

$$
\mathbf{F}_{p} \otimes_{T_{f} R} N \cong \mathbf{F}_{p} \otimes_{T_{f} H}\left(T_{f} H \otimes_{T_{f} R} N\right) .
$$

This gives rise to a composition of functors spectral sequence

$$
E_{i, j}^{2}=\operatorname{Tor}_{i}^{T_{f} H}\left(\mathbf{F}_{p}, \operatorname{Tor}_{j}^{T_{f} R}\left(T_{f} H, N\right)\right) \Rightarrow \operatorname{Tor}_{i+j}^{T_{f} R}\left(\mathbf{F}_{p}, N\right) .
$$

We will show that for $N=T_{f}(M)$ this spectral sequence has $E_{i, j}^{2}=0$ for $(i, j) \neq$ $(0,0)$.

Note first of all that the groups $\operatorname{Tor}_{j}^{R}(H, M)$ vanish for $j>0$ because $M$ is free as an $R$-module (here $R$ acts on $H$ via $f$ ). By 3.5 , $\operatorname{Tor}_{j}^{T_{f} R}\left(T_{f} H, T_{f} M\right)=0$ for $j>0$. Now consider the object

$$
U=\operatorname{Tor}_{0}^{T_{f} R}\left(T_{f} H, T_{f} M\right)=T_{f}\left(H \otimes_{R} M\right)
$$

as a module over $T_{f}(H)$. By $[19,3.8 .6]$ and $[19,3.9], T_{f}(H)$ is isomorphic to a direct product $\prod_{\alpha} H(\alpha)$ of copies of $H$ indexed by the (finite) collection of $\mathcal{K}$-maps $\alpha: H \rightarrow H$ with $\alpha \cdot f=f$. Under this isomorphism, the map $\iota^{b}: T_{f}(H) \rightarrow \mathbf{F}_{p}$ is obtained by composing projection on the component $H(\iota)$ with the unique ring homomorphism $H(\iota)=H \rightarrow \mathbf{F}_{p}$. Since $H(\iota)=H$ is flat (in fact projective) over $T_{f}(H)$, there are natural isomorphisms

$$
\operatorname{Tor}_{i}^{T_{f} H}\left(\mathbf{F}_{p}, U\right) \cong \operatorname{Tor}_{i}^{H}\left(\mathbf{F}_{p}, H \otimes_{T_{f} H} U\right), \quad i \geq 0 .
$$

The projection $T_{f}(H) \rightarrow H(\iota)$ can alternatively be interpreted as the natural map $T_{f}(H) \rightarrow T_{\iota}(H)$. This implies that there are isomorphisms

$$
\begin{aligned}
H \otimes_{T_{f} H} U & \cong T_{\iota} H \otimes_{T_{f} H} T_{f} H \otimes_{T_{f} R} T_{f} R \otimes_{T R} T M \\
& \cong T_{\iota} H \otimes_{T H}\left(T H \otimes_{T R} T M\right) \\
& \cong T_{\iota H} \otimes_{T H} T\left(H \otimes_{R} M\right) \\
& \cong T_{\iota}\left(H \otimes_{R} M\right)
\end{aligned}
$$

where we have used the fact that $T$ is exact and preserves tensor products to give the isomorphism $T H \otimes_{T R} T M \cong T\left(H \otimes_{R} M\right)$ (cf. 3.5). The desired vanishing now follows from the fact that if $N$ is any object of $\mathcal{U}(H)$, in particular $H \otimes_{R} M$, then $T_{\iota}(N)$ is free as a module over $T_{\iota}(H) \cong H[10,2.4][16,4.5]$.

\section{Rings OF INVARIANTS AND $T$}

In order to deduce Theorem 1.4 from Theorem 1.1 we have to find a connection between rings of invariants, algebras over $\mathcal{A}_{p}$, and the functor $T$. This is provided by the following construction. 
5.1 Definition. Suppose that $V$ is a finite dimensional vector space over $\mathbf{F}_{p}$. The enhanced symmetric algebra $\mathbf{S}\left(V^{\#}\right)$ is the unstable algebra over $\mathcal{A}_{p}$ freely generated as a commutative algebra by the elements of $V^{\#}$, which are considered to have grading two. The action of $\mathcal{A}_{p}$ on $\mathbf{S}\left(V^{\#}\right)$ is the unique one allowed by the usual unstable algebra conditions.

5.2 Remark. The action of $\mathcal{A}_{p}$ on $\mathbf{S}\left(V^{\#}\right)$ can be described explicitly as follows. If $p$ is odd and $x \in V^{\#}=\mathbf{S}\left(V^{\#}\right)^{2}$, then $\mathcal{P}^{1}(x)=x^{p} \in \mathbf{S}\left(V^{\#}\right)^{2 p}, \beta(x)=0$, and $\mathcal{P}^{i}(x)=0$ for $i>1 ; \mathcal{A}_{p}$ acts on products of two-dimensional classes by the Cartan formula. The same formulas work for $p=2$ if $\beta$ is interpreted as $\mathrm{Sq}^{1}$ and $\mathcal{P}^{i}$ as $\mathrm{Sq}^{2 i}$. The algebra $\mathbf{S}\left(V^{\#}\right)$ is isomorphic as an element of $\mathcal{K}$ to the cohomology ring of a product of copies of $C P^{\infty}$, where the number of factors in the product is the dimension of $V ; \mathbf{S}\left(V^{\#}\right)$ is also isomorphic to the subalgebra of $\mathrm{H}^{*}\left(\mathrm{~B} V ; \mathbf{F}_{p}\right)$ generated by the Bocksteins of one-dimensional cohomology classes. In particular $\mathbf{S}\left(V^{\#}\right)$ is functorial in $V$, and is isomorphic as an algebra to $\operatorname{Sym}\left(V^{\#}\right)$; it differs from $\operatorname{Sym}\left(V^{\#}\right)$ only in that it is explicitly graded and has a specified action of $\mathcal{A}_{p}$.

5.2 Lemma. Suppose that $E$ and $V$ are finite-dimensional vector spaces over $\mathbf{F}_{p}$. Then there is a natural bijection

$$
\operatorname{Hom}_{\mathcal{K}}\left(\mathbf{S}\left(V^{\#}\right), \mathrm{H}^{*}\left(\mathrm{~B} E ; \mathbf{F}_{p}\right)\right) \cong \operatorname{Hom}(E, V)
$$

and a natural $\mathcal{K}$-isomorphism between $T_{E} \mathbf{S}\left(V^{\#}\right)$ and $\mathbf{S}\left(V^{\#}\right)^{\operatorname{Hom}(E, V)}$.

Remark. The notation $\mathbf{S}\left(V^{\#}\right)^{\operatorname{Hom}(E, V)}$ from 5.3 denotes the collection of set-maps from $\operatorname{Hom}(E, V)$ to $\mathbf{S}\left(V^{\#}\right)$, with pointwise ring operations; equivalently, this is a product of copies of $\mathbf{S}\left(V^{\#}\right)$ indexed by the elements of $\operatorname{Hom}(E, V)$. Under the isomorphism of 5.3 the action of $\operatorname{Aut}(V)$ on $T_{E} \mathbf{S}\left(V^{\#}\right)$ corresponds to the diagonal action of $\operatorname{Aut}(V)$ on $\mathbf{S}\left(V^{\#}\right)^{\operatorname{Hom}(E, V)}$, in other words, the action which gives $g \in$ $\operatorname{Aut}(V), \phi \in \operatorname{Hom}(E, V)$, and $\alpha: \operatorname{Hom}(E, V) \rightarrow \mathbf{S}\left(V^{\#}\right)$ has $(g \cdot \alpha)(\phi)=g \alpha\left(g^{-1} \phi\right)$.

The first statement in 5.3 is related to the calcualtion of Adams, Gunawardena and Miller in [1, p. 438] but simpler, because it involves morphisms of unstable algebras (not modules) over the Steenrod algebra.

Proof of 5.2. Let $H=H^{*}$ denote $\mathrm{H}^{*}\left(\mathrm{~B} E ; \mathbf{F}_{p}\right)$. The calculation of $\operatorname{Hom}_{K}\left(\mathbf{S}\left(V^{\#}\right), H\right)$ is as follows. Any $f \in \operatorname{Hom}_{K}\left(\mathbf{S}\left(V^{\#}\right), H\right)$ is an algebra map and so is determined by its effect on the copy of $V^{\#}$ in dimension 2. Clearly $f\left(V^{\#}\right)$ lies in the kernel of the Bockstein map $\beta: H^{2} \rightarrow H^{3}$. Since $\tilde{\mathrm{H}}^{*}(\mathrm{~B} E ; \mathbb{Z})$ is of exponent $p$, this kernel is isomorphic to $H^{1}=E^{\#}$ via $\beta: H^{1} \rightarrow H^{2}$ (cf. $[19,1.5]$ ). Thus any such $f$ gives a map $V^{\#} \rightarrow E^{\#}$ or equivalently a map $E \rightarrow V$. It is now easy to check that any homomorphism $V^{\#} \rightarrow E^{\#}$ arises from a unique $f$.

For any object $R$ of $\mathcal{K}$ there is a natural map $R \rightarrow T_{E}(R)$ adjoint to the morphism $R \otimes_{\mathbf{F}_{p}} H^{*} \rightarrow R \otimes_{\mathbf{F}_{p}} \mathbf{F}_{p} \cong R$ induced by the unique $K$-map $H \rightarrow \mathbf{F}_{p}$. For any $f: R \rightarrow H$ there is a composite map $\epsilon_{f}: R \rightarrow T_{E} R \rightarrow T_{E, f} R$. The last statement in the lemma follows from the fact that for any $f: \mathbf{S}\left(V^{\#}\right) \rightarrow H$ the map $\epsilon_{f}: \mathbf{S}\left(V^{\#}\right) \rightarrow T_{E, f} \mathbf{S}\left(V^{\#}\right)$ is an isomorphism. The most economical way to obtain this is from a geometric theorem of Lannes [19, 9.6] [15]. Let $G$ denote the circle group $S^{1}=S O(2)$. The algebra $\mathbf{S}\left(V^{\#}\right)$ is isomorphic to $\mathrm{H}^{*}\left(\mathrm{~B} G^{d} ; \mathbf{F}_{p}\right)$, where $d=\operatorname{dim} V$ (cf. 5.2). By Lannes' theorem, a $\mathcal{K}$-map $f: \mathbf{S}\left(V^{\#}\right) \rightarrow H$ corresponds to a homomorphism $h(f): E \rightarrow S G^{d}$, and $T_{f} \mathbf{S}\left(V^{\#}\right)$ is then naturally isomorphic to the cohomology of the classifying space of the centralizer in $G^{d}$ of the image 
of $h(f)$. Since $G^{d}$ is abelian, this centralizer is $G^{d}$ itself, and the cohomology of its classifying space is again $\mathbf{S}\left(V^{\#}\right)$.

Proof of 1.4. Let $S=\mathbf{S}\left(V^{\#}\right)$ and $R=S^{W}$. Suppose that $E$ is an elementary abelian $p$-group. It follows directly from the exactness of $T$ that there is a $\mathcal{K}$ isomorphism $T_{E} R=T_{E}\left(S^{W}\right) \cong\left(T_{E} S\right)^{W}[19,3.9 .5]$. By 5.3, this gives isomorphisms

$$
\begin{aligned}
T_{E}(R)=T_{E}\left(S^{W}\right) & \cong \operatorname{Map}(\operatorname{Hom}(E, V), S)^{W} \\
& \cong \operatorname{Map}_{W}(\operatorname{Hom}(E, V) S) \\
& \cong \prod_{\{\phi\}} S^{W_{\phi}} .
\end{aligned}
$$

Here the product in the third line is taken over the set of orbits $\{\phi\}$ of the action of $W$ on $\operatorname{Hom}(E, V)$, and $W_{\phi}$ denotes the isotropy subgroup in $W$ of an orbit representative $\phi$. To pass from the second to the third line we have used the fact that if $A$ and $B$ are $W$-sets and $W$ acts transitively on $A$, there is an isomorphism $\operatorname{Map}_{W}(A, B) \cong B^{W_{a}}$, where $a$ is any element of the orbit $a$.

It follows from the dimension 0 part of 5.4 that $\mathcal{K}$-maps $f: R \rightarrow \mathrm{H}^{*}\left(\mathrm{~B} E ; \mathbf{F}_{p}\right)$ (equivalently ring homomorphisms $T^{0} R \rightarrow \mathbf{F}_{p}$ ) are in bijective correspondence with orbits $\{\phi\}$ of the action of $W$ on $\operatorname{Hom}(E, V)$. Moreover, for any such $f$, $T_{f}(R)$ is isomorphic to $S^{W_{\phi}}$. Let $U$ be the chosen subset of $V, E$ the linear span of $U$, and $\phi: E \rightarrow V$ the inclusion. The $W_{U}$ (the subgroup of $W$ consisting of elements which fix $U$ pointwise) is equal to $W_{\phi}$ (the subgroup of $W$ consisting of elements which under composition leave the map $\phi$ unchanged). Let $f: R \rightarrow$ $\mathrm{H}^{*}\left(\mathrm{~B} E ; \mathbf{F}_{p}\right)$ correspond to $\{\phi\}$. Since $R$ is a polynomial algebra by assumption (finitely generated for elementary transcendence degree reasons), it follows from 1.1 that $T_{f}(R) \cong S^{W_{\phi}} \cong S^{W_{U}} \cong \operatorname{Sym}\left(V^{\#}\right)^{W_{U}}$ is also a polynomial algebra.

\section{A UNIVERSAL EXAMPLE WITH NON-REFLECTION STABILIZERS}

We provide a "universal" example to satisfy the promise of Example 1.10.

6.1 Example. Let $\Sigma$ be the rank 3 free abelian subgroup of $G L(5, \mathbb{Z})$ generated by the matrices

$$
\left(\begin{array}{lllll}
1 & 0 & 1 & 0 & 0 \\
0 & 1 & 1 & 0 & 0 \\
0 & 0 & 1 & 0 & 0 \\
0 & 0 & 0 & 1 & 0 \\
0 & 0 & 0 & 0 & 1
\end{array}\right), \quad\left(\begin{array}{lllll}
1 & 0 & 0 & 1 & 0 \\
0 & 1 & 0 & 0 & 0 \\
0 & 0 & 1 & 0 & 0 \\
0 & 0 & 0 & 1 & 0 \\
0 & 0 & 0 & 0 & 1
\end{array}\right), \quad\left(\begin{array}{lllll}
1 & 0 & 0 & 0 & 0 \\
0 & 1 & 0 & 0 & 1 \\
0 & 0 & 1 & 0 & 0 \\
0 & 0 & 0 & 1 & 0 \\
0 & 0 & 0 & 0 & 1
\end{array}\right),
$$

denoted $r, s$ and $t$ respectively. The stabilizer of $(0,0,1,-1,-1)^{T}$ in $\Sigma$ is the subgroup generated by the product $z=r s t$. Then

$$
z=\left(\begin{array}{lllll}
1 & 0 & 1 & 1 & 0 \\
0 & 1 & 1 & 0 & 1 \\
0 & 0 & 1 & 0 & 0 \\
0 & 0 & 0 & 1 & 0 \\
0 & 0 & 0 & 0 & 1
\end{array}\right)
$$

and so $z-\operatorname{Id}$ has rank 2 . Let $\rho: G L(5, \mathbb{Z}) \rightarrow G L\left(5, \mathbf{F}_{p}\right)$ be the $\bmod p$ reduction map. Then the images of $\{r, s, t\}$ are reflections of order $p$, and $\rho(\Sigma)$ is an elementary abelian $p$-group of rank 3 . In this image, the stabilizer of the reduction $\bmod p$ of 
$(0,0,1,-1,-1)^{T}$ is the subgroup of order $p$ generated by $\rho(z)$ and thus contains no reflections.

Proof. The verification is a straightforward calculation. The matrices have been chosen so that multiplication of the matrices corresponds to addition of the upper right $2 \times 3$ blocks - if $A$ and $B$ are matrices which are zero off this block, then $A B=0$ and $(I+A)(I+B)=(I+B)(I+A)$. Hence the group $\Sigma$ is commutative and isomorphic to the free abelian group of rank 3 , and $\rho(\Sigma)$ is a rank 3 elementary abelian $p$-group. Clearly, $r^{a}\left(s^{b}\left(t^{c}\left((0,0,1,-1,-1)^{T}\right)\right)\right)=(a-b, a-c, 1,-1,-1)^{T}$, so the stabilizer of $(0,0,1,-1,-1)^{T}$ consists of the elements $r^{a} s^{b} t^{c}$ with $a=b$ and $a=c$. This is the group generated by $z^{a}$, where $z=r s t$. An identical calculation works $\bmod p$.

\section{REFERENCES}

1. J. F. Adams, J. H. Gunawardena, and H. Miller, The Segal conjecture for elementary abelian p-groups, Topology 24 (1985), 435-460. MR 87m:55026

2. D. J. Benson, Polynomial Invariants of Finite Groups, Cambridge University Press, 1994.

3. A. Borel, Groupes linéaires algébriques, Annals of Mathematics 64(2) (195), 20-82. MR 19:1195k

4. A. Borel, Sous groupes commutatifs et torsion des groupes de Lie compacts connexes, Tôhoku Math. J. 13(2) (1961), 216-240. MR 26:5094

5. N. Bourbaki, Groupes et algèbres de Lie, Ch. 4, 5 et 6., Masson, Paris, 1981. MR 39:1590

6. H.E.A. Campbell, J.P. Hughes, and J. Shank, Preliminary notes on rigid reflection groups, preprint (1995).

7. C. Chevalley, Invariants of finite groups generated by reflections., Amer. J. Math. 67 (1955), 778-782. MR 17:345d

8. H. S. M. Coxeter, Discrete groups generated by reflections., Ann. Math. 35 (1934), 588-621.

9. W.G. Dwyer and C.W. Wilkerson, Torsion in the cohomology of classifying spaces, hour address by Clarence Wilkerson, Holyoke College (1994).

10. W.G. Dwyer and C.W. Wilkerson, Smith Theory and the functor T, Comm. Math. Helv. 66 (1991), 1-17. MR 92i:55006

11. W. G. Dwyer and C. W. Wilkerson, Homotopy fixed point methods for Lie groups and finite loop spaces, Annals of Math. 139 (1994), 395-442. MR 95e:55019

12. W. G. Dwyer and C. W. Wilkerson, The center of a p-compact group, Contemporary Mathematics 181, Proceedings of the 1993 Cech Conference (Northeastern Univ.), Amer. Math. Soc., Providence, 1995, pp. 119-157. MR 96a:55024

13. D. Eisenbud, Commutative Algebra. With a View Toward Algebraic Geometry, Grad. Texts in Math 150, Springer, 1995. MR 97a:13001

14. G. Kemper and G. Malle, The finite irreducible linear groups with polynomial rings of invariants, Universität Heidelberg preprint 96-38 (August 1996).

15. J. Lannes, Cohomology of groups and function spaces, preprint (1986).

16. J. Lannes, Sur les espaces fonctionnels dont la source est le classifiant d'un p-groupe abélien élémentaire, Publ. I.H.E.S. 75 (1992), 135-244. MR 93j:55019

17. H. Matsumura, Commutative ring theory, Cambridge University Press, 1986. MR 88h:13001

18. H. Nakajima, Regular rings of invariants of unipotent groups, J. of Algebra 85(2) (1983), 253-286. MR 85f:20038

19. Lionel Schwartz, Unstable Modules over the Steenrod Algebra and Sullivan's Fixed Point Set Conjecture, University of Chicago Press, 1994. MR 95d:55017

20. J.-P. Serre, Groupes finis d'automorphismes d'anneaux locaux réguliers, Colloq. d'Alg. E.N.S. (1967).

21. G. C. Shephard and J. A. Todd, Finite unitary reflection groups, Canad. J. Math. 6 (1954), 274-304. MR 15:6000b 
22. R. Steinberg, Differential equations invariant under finite reflection groups, A. M. S. Transactions 112 (1964), 392-400. MR 29:4807

23. H. Toda, Cohomology mod 3 of the classifying space $B F_{4}$ of the exceptional group $F_{4}$, J. Math. Kyoto Univ. 13 (1973), 97-115. MR 47:9619

Department of Mathematics, University of Notre Dame, Notre Dame, Indiana 46556

E-mail address: dwyer.10nd.edu

Department of Mathematics, Purdue University, West Lafayette, Indiana 47907

E-mail address: wilker@math.purdue.edu 